\title{
Research Article \\ Optical Design with Narrow-Band Imaging for a Capsule Endoscope
}

\author{
Chih-Ta Yen $\mathbb{D}^{1}{ }^{1}$ Zong-Wei Lai, ${ }^{2}$ Yu-Ting Lin, ${ }^{1}$ and Hsu-Chih Cheng $\mathbb{D}^{2}$ \\ ${ }^{1}$ Department of Electrical Engineering, National Formosa University, Yunlin, Taiwan \\ ${ }^{2}$ Department of Electro-Optics Engineering, National Formosa University, Yunlin, Taiwan \\ Correspondence should be addressed to Chih-Ta Yen; chihtayen@gmail.com
}

Received 28 November 2016; Revised 22 April 2017; Accepted 19 September 2017; Published 10 January 2018

Academic Editor: Valentina Camomilla

Copyright (C) 2018 Chih-Ta Yen et al. This is an open access article distributed under the Creative Commons Attribution License, which permits unrestricted use, distribution, and reproduction in any medium, provided the original work is properly cited.

\begin{abstract}
The study proposes narrow-band imaging (NBI) lens design of $415 \mathrm{~nm}$ and $540 \mathrm{~nm}$ of a capsule endoscope (CE). The researches show that in terms of the rate of accuracy in detecting and screening neoplastic and nonneoplastic intestinal lesions, the NBI system outperformed that of traditional endoscopes and rivaled that of chromoendoscopes. In the proposed NBI CE optical system, the simulation result shows the field of view (FOV) was $109.8^{\circ}$; the modulation transfer function (MTF) could achieve $12.5 \%$ at $285 \mathrm{lp} / \mathrm{mm}$ and $34.1 \%$ at $144 \mathrm{lp} / \mathrm{mm}$. The relative illumination reaches more than $60 \%$, and the system total length was less than $4 \mathrm{~mm}$. Finally, this design provides high-quality images for a 300-megapixel 1/4" CMOS image sensor with a pixel size of $1.75 \mu \mathrm{m}$.
\end{abstract}

\section{Introduction}

Modern-day eating habits have increased the burden on the digestive systems of most people, and gastrointestinal diseases have emerged as a widespread problem. Some of the conventional gastrointestinal examination methods include gastroscopy and colonoscopy. The former examines areas such as the esophagus, stomach, duodenum, and the front section of the small intestine, whereas the latter (in which the examination device is inserted through the anus) examines areas such as the rectum, the large intestine, and the end section of the small intestine. The small intestine, which makes up $75 \%$ of the human gastrointestinal tract, is the site where symptoms are most difficult to spot. Because conventional endoscopes are invasive, most people fear to visit the hospital; as a result, their diseases worsen. However, with advances in medical technology in recent years, a new tool has been developed to inspect intestinal diseases; it is called the "capsule endoscope" (CE) [1].

After the patient has swallowed a CE, it is pushed through the patient's digestive tract by the mechanism of enterogastric peristalsis. The CE is equipped with a lighting source, an optical lens, and an image sensor. By using such devices, it captures images of the human digestive organs; image data are subsequently sent through radiofrequency (RF) and microwave signals to an external storage device worn by the patient for data storage. The CE procedure, in which the $\mathrm{CE}$ enters from the esophagus to the stomach and later to the small intestine, takes approximately six to eight hours to complete. A CE takes color photographs at a rate of two photographs per second, producing roughly $50,000-60,000$ photographs during the process. The $\mathrm{CE}$ is later excreted naturally, completing the inspection process. Finally, computers are used to read image data extracted from the storage device memory, and data are made into dynamic videos viewed by the physicians who diagnose the diseases. Lewis and Swain indicated that the use of CEs can improve disease detection rate as well as lowering the discomfort experienced by patients [2]. This showed that CE examination is a more accurate and beneficial method for inspecting small intestinal diseases than traditional methods.

In 1999, Given Imaging designed a CE prototype, and in August 2011, it developed an official CE approved by the FDA; the product was subsequently released in the US market. Currently, Olympus is the major domestic CE brand in Japan. The company's patent [3] presents an optical system 
design similar to that in the study of Ramsden eyepieces [4]. By considering the size limit of the CE, they proposed a single plastic lens with an aspherical surface composed of two flat convex lenses, thus simplifying the structure and meeting the volume limits. This design makes the optical system smaller by reducing the number of lenses and shortening the optical path. However, when the number of lenses is reduced, the quality of the image also decreases and the distortion increases. The lens parameters of the proposed patent are as follows: effective focal length, $f=1 \mathrm{~mm}$; field of view angle $=85^{\circ}-110^{\circ}$; optical total length, $L<2.4 \mathrm{~mm}$; image plane radius $<1 \mathrm{~mm}$; and depth of field $(\mathrm{DOF})=5.45-$ $30 \mathrm{~mm}$. Therefore, the lens can be used only on CCD or CMOS image sensors smaller than $1 / 10^{\prime \prime}$. Seo et al. proposed an MEMS-based liquid lens, which can improve image quality by using the autofocus and zoom-in functions. However, a bias voltage of up to $30 \mathrm{~V}$ is required to consider the actual characteristics of $\mathrm{CE}$, and hence, the commercialization of CE systems is difficult [5].

Chen [6] noted that the use of a conical optical reflector and a transparent, ring-shaped view window in the CE design served the primary purpose of overcoming CE mechanism limitations. Early CEs demonstrated astigmatism defects in focus. By increasing the number of focal lens groups and changing the location of the conical optical reflector, Chen corrected the astigmatism of the conical optical reflector and enhanced the contact-based CE image quality [6]. In that study, the CE captured images primarily from its sides and had an FOV of $65.08^{\circ}$. The new lens group, which features an $\mathrm{F} / \#$ of 4.2 , generated a maximum MTF of $36 \%$ at a spatial frequency of $100 \mathrm{lp} / \mathrm{mm}$.

Ou-Yang and Jeng introduced a new-generation radial imaging capsule endoscope (RICE) that achieved ring-field scanning by using a conical lens; a transparent, ring-shaped view window; and an optical focusing system [7]. Because stray light lowered the lighting uniformity of the RICE, various improvements were made to the RICE lighting system, including changing the locations of LEDs, cutting off a section of the tip of a conical lens, and employing a multilayer coating method, after which lighting uniformity was enhanced from $12 \%$ to $69 \%$. In addition, a clinical, in vivo experiment was administered to verify the effectiveness of the RICE. The device was fed to a pig. Small images of the pig's small intestine were stitched together to form composite images. Pearson's correlation coefficients verified that the images effectively represented complex intestinal environments. Tseng et al. [8] proposed a color multiplexing capsule endoscope (CMCE), which adopted a color multiplexing method to enable its $\mathrm{x}$-cube prism to simultaneously record frontal images as well as those on the two sides [8]. The images were presented in three different colors, namely, green, red, and blue. Each side of the CMCE had a viewing angle of $70^{\circ}$, and images were combined to produce a full viewing angle of $210^{\circ}$. This allowed the CE to produce favorable image quality with a miniaturized device.

CEs can be classified into three types. In this study, the traditional frontal image-based CE model was upgraded by using CE structures proposed by Mang et al. [9] and Tang et al. [10]. The former reduced distortion without adding

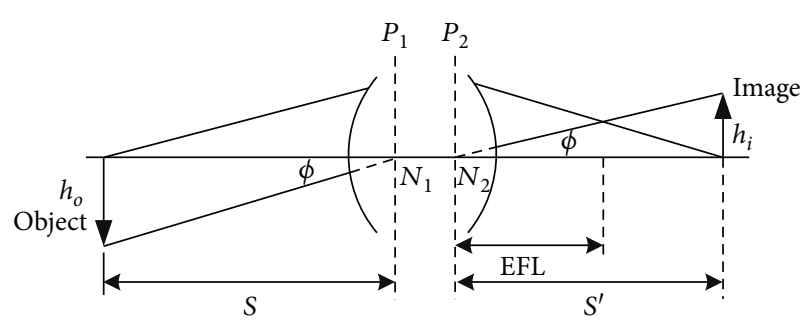

FIgURE 1: Schematic of the image and object in relation to each other.

TABle 1: Design indicators.

\begin{tabular}{lc}
\hline Field of view (FOV) & $110^{\circ}$ \\
F/\# & $1 / 2.8$ \\
Half of the image height & $2.3 \mathrm{~mm}$ \\
Effective focal length (EFL) & $2 \mathrm{~mm}$ \\
Blake focal length (BFL) & $1 \mathrm{~mm}$ \\
Total length & $4 \mathrm{~mm}$ \\
Relative illumination & $>50 \%$ \\
Distortion & $<20 \%$ \\
\hline
\end{tabular}

additional lenses (to avoid increasing the size of the CE) by making the CE optical protective mask into a lens and turning object planes into flat planes, whereas the latter used fewer aspheric lens than were used in [9] and enhanced lens groups to facilitate CE production.

\section{Research Methods}

This section presents the overall design process of a CE optical system; optical system design methods can be divided into the following steps: (1) determining the optical uses of the system, (2) setting design indicators, (3) selecting initial structure, (4) correcting optical aberrations, and (5) testing and inspecting performance.

2.1. Setting Specifications. Because the CE must travel through human organs, the aperture and effective focal lens (EFL) values of its cameras cannot be overly large. Therefore, this study set the EFL, back focal length (BFL), and F/\# at $2 \mathrm{~mm}, 1 \mathrm{~mm}$, and 2.8 , respectively. In addition, because the overall CE size must be controlled, the total length of a camera lens was set at approximately $4 \mathrm{~mm}$.

The CMOS selected in this study was an OV3640 model, which featured a complementary metal-oxide-semiconductor (CMOS) image sensor, an effective pixel arrangement of $2048 \times 1536$, a total resolution of approximately 300 million pixels (size of single pixel $=1.75 \mu \mathrm{m} \times 1.75 \mu \mathrm{m}$ ), and a semiimage height of $2.3 \mathrm{~mm}$. Using Figure 1, the object distance, image distance, and FOV required were derived.

The Gaussian imaging equation is as follows:

$$
\frac{1}{s^{\prime}}-\frac{1}{s}=\frac{1}{f} \text {. }
$$


TABLE 2: Comparison between CMOS selection in this study and that presented in existent literature.

\begin{tabular}{lccr}
\hline & Ou-Yang Mang & Lilai Tang & Research selection \\
\hline Active array & $256 \times 256$ & $1600 \times 1200$ & $2048 \times 1536$ \\
Pixel size & $10 \mu \mathrm{m} \times 10 \mu \mathrm{m}$ & $2.2 \mu \mathrm{m} \times 2.2 \mu \mathrm{m}$ & $1.75 \mu \mathrm{m} \times 1.75 \mu \mathrm{m}$ \\
Image area & $2.7 \mathrm{~mm} \times 2.7 \mathrm{~mm}$ & $3.6 \mathrm{~mm} \times 2.7 \mathrm{~mm}$ & $3.6 \mathrm{~mm} \times 2.7 \mathrm{~mm}$ \\
Length of the diagonal line & $3.8 \mathrm{~mm}$ & $2.2 \mathrm{~mm}$ & $2.26 \mathrm{~mm}$ \\
\hline
\end{tabular}

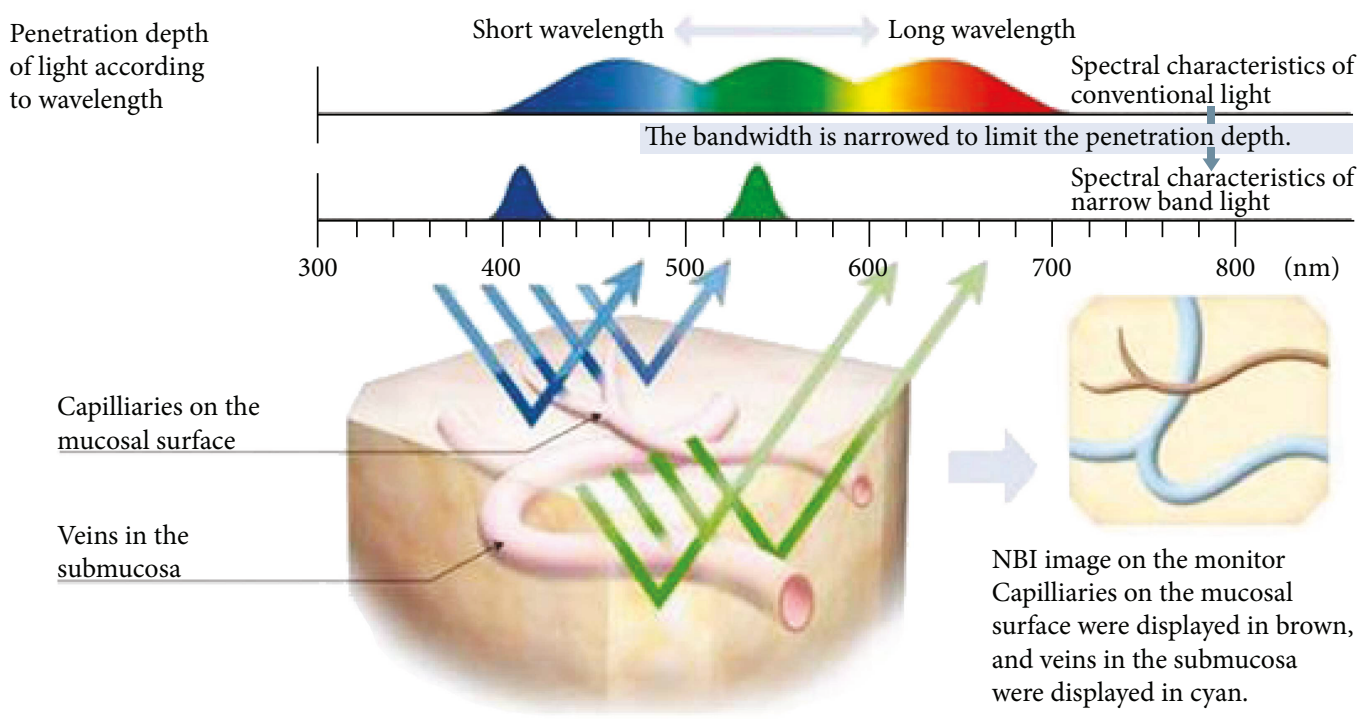

FIGURE 2: Narrow-band imaging [17]. follows:

The image longitudinal magnification equation is as

$$
m=\frac{h_{i}}{h_{o}}=\frac{s^{\prime}}{s}
$$

By multiplying the two sides of (2) by $s$, the following equation is derived:

$$
s=f\left(\frac{1}{m}-1\right)
$$

where $f, s$, and $s^{\prime}$ are the EFL, object distance, and image distance, respectively.

Because the diameter of the small intestine is approximately $25 \mathrm{~mm}$ [11] and the length of an intestinal villus is approximately $1.25 \mathrm{~mm}$, object height was set at approximately $11.25 \mathrm{~mm}$. In addition, because one-half of the length of the diagonal line joining the image height and the object was approximately $2.3 \mathrm{~mm}, h_{o}$ and $h_{i}$ were set at 11.25 and $2.3 \mathrm{~mm}$, respectively. Using (3), the $m, s$, and $s^{\prime}$ were calculated as $0.2,8$, and 1.6 , respectively. The FOV was determined by solving the equations $\tan \phi=h_{o} / s$ and $\phi=\tan ^{-1}\left(h_{o} / s\right)$, that is, $\phi=54.6^{\circ}$. Therefore, FOV was calculated to be approximately $109.2^{\circ}$.

The design indicators of this study are shown in Table 1 [12].
2.2. Image Sensor. The human body structure was considered for calculating the optical system specifications in the study (Table 1). The CMOS model OV3640 was selected in this study, and its specifications are as follows: an effective pixel arrangement of $2048 \times 1536$ and a total resolution of approximately 300 million pixels (size of single pix$\mathrm{el}=1.75 \times 1.75 \mu \mathrm{m})$. A comparison of the specifications of different CMOS image sensors used in optical CE systems is presented in Table 2 [13].

When evaluating the quality of a camera lens, the most essential criteria are its resolution and sharpness. At a low spatial frequency, a camera lens that produces high MTF indicates superior contrast, that is, clearer image outline; similarly, at a high spatial frequency, a camera lens that produces high MTF signifies more favorable sharpness, that is, clearer subtle details. The spatial frequency that can be analyzed by a CMOS image sensor is related to the pixel size of the images. By using (4), the Nyquist frequency can be obtained [14].

$$
\mathrm{MTF}=\frac{1}{2 \times \text { pixel size }(\mathrm{mm})} .
$$

Given that the size of a single pixel was $1.75 \mu \mathrm{m}$, the maximum spatial frequency was calculated to be $285.7 \mathrm{lp} /$ $\mathrm{mm}$. To obtain favorable image quality, the spatial frequency should be $10 \%$ or higher at an MTF of $285 \mathrm{lp} / \mathrm{mm}$.

2.3. Narrow-Band Imaging (NBI). The accuracy of traditional white-light endoscopy in identifying and diagnosing 
neoplastic and nonneoplastic lesions is approximately $70 \%$, whereas that of chromoendoscopy and magnifying endoscopy is between $90 \%$ and $95 \%$. Because chromoendoscopy uses a photographic developer to highlight subtle lesion changes, it requires dyes to be sprayed in advance, which increases treatment time. Virtual chromoendoscopes have thus been developed. Of the various imaging methods adopted by virtual chromoendoscopes, the narrow-band imaging method is the most common. Narrow-band technology was developed based on a physical theory; that is, the longer the wavelength, the better its penetration rate. In the visible light spectrum, red light wave has the longest wavelength, followed by green and blue light waves. When two light sources of different wavelengths [i.e., blue (wavelength: $415 \mathrm{~nm}$ ) and green (wavelength: $540 \mathrm{~nm}$ )] illuminate the intestine, the blue and green light waves penetrate shallower and deeper layers of the gastrointestinal tract mucosa, respectively (Figure 2). Because capillaries (brown in color) in the epithelial mucosal tissues are detectable by blue light waves and because blood vessels (cyan in color) in the subcutaneous mucosal tissues are detectable by green light waves, they assist observers in identifying epithelial and subcutaneous mucosal tissue lesions. Sano et al. [15] and Machida et al. [16] both showed that in terms of the rate of accuracy in detecting and screening neoplastic and nonneoplastic intestinal lesions, the NBI system outperformed that of traditional endoscopes and rivaled that of chromoendoscopes.

The CE design flow chart of this study is shown in Figure 3.

This section presents the overall design process of the CE optical system; optical system design methods can be divided into the following steps: (1) determining the optical uses of the system, (2) setting design indicators, (3) selecting initial structure, (4) correcting optical aberrations, and (5) testing and inspecting performance.

\section{Experiment Results}

3.1. Literature Review. The CE model used in this study was developed by referring to CE-related literature written by Mang et al. [9] and Tang et al. [10]. Their CE designs are presented as follows:

(1) Mang et al. [9]

Figure 4(a) shows the camera lens structure presented by Mang et al. [9], which produced an FOV of $86^{\circ}$ and an MTF value of $26 \%$ at $100 \mathrm{lp} / \mathrm{mm}$ (Figure 4(b)).

(2) Tang et al. [10]

Figure 5(a) shows the design results of Tang et al. [10], which produced an FOV of up to $105^{\circ}$, an MTF value of $40 \%$ at $45 \mathrm{lp} / \mathrm{mm}$ (Figure 5(b)), and a distortion of less than $15 \%$.

From the 3D layout diagrams of the lenses used in the two studies, we inferred that although both studies adopted 3-lens designs, their MTF values can be further improved.

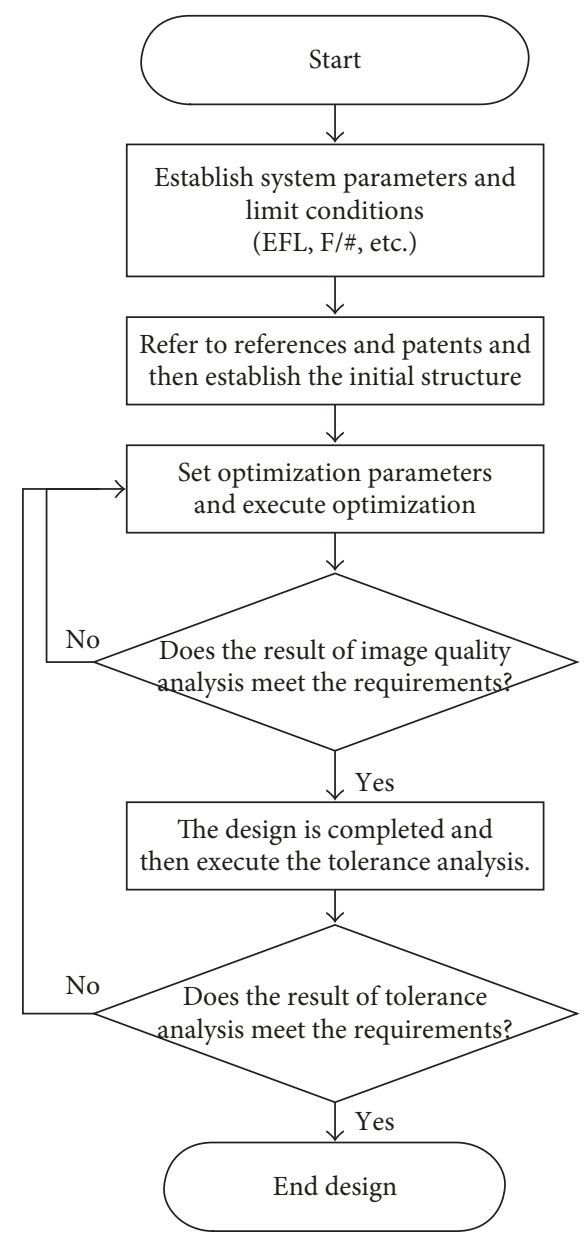

Figure 3: CE design flow chart.

For example, with the advances in imaging technology, CMOS resolutions have been increasing and different lens materials have been introduced. Therefore, in this study, an additional lens was added to the lens group design to enhance image quality (under the constraint of CE size restrictions) and make the lens group applicable to 300-megapixel CMOS designs.

3.2. Initial Structural Design. The F/\# input was set at 2.8. Concerning the FOV, because the CMOS produced an image height of $2.3 \mathrm{~mm}$, real image height was selected for the FOV and inputs $0.0,0.5,1.0,1.5,2.0$, and 2.3 were entered. The wavelengths used were set at 415 and $540 \mathrm{~nm}$ considering the use of narrow-band technology. Table 3 and Figure 6 show the lens parameters and initial structure, respectively, which reveal that the focus of the image plane had a room for improvement.

Figure 7 shows the MTF diagram of the initial structure, in which the horizontal axis signifies the spatial frequency and the vertical axis denotes how ideal the images were (each image was measured as a percentage of ideal images). The different colored curves represent the MTG under different FOVs. The diagram shows that at the maximum spatial frequency (i.e., $285 \mathrm{lp} / \mathrm{mm}$ ), MTF was $1.5 \%$. 


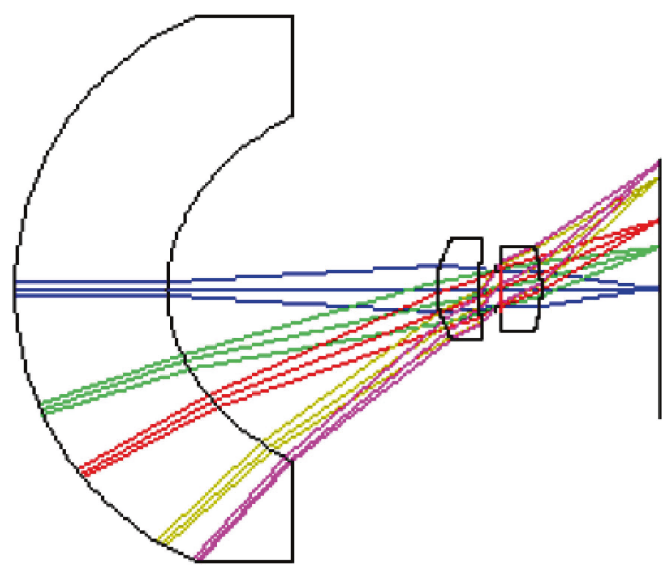

(a)

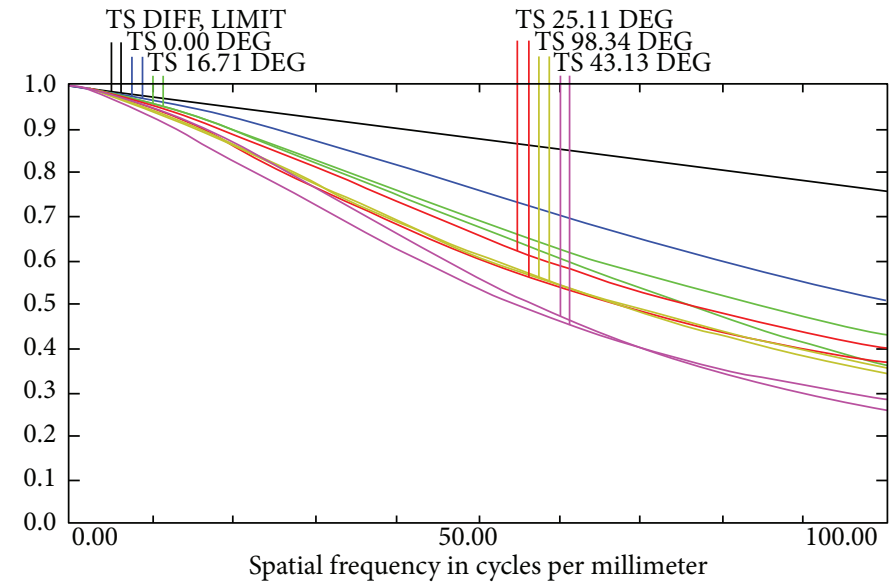

(b)

FIGURE 4: (a) 3D layout used in existent literature. (b) MTF values generated in existent literature.

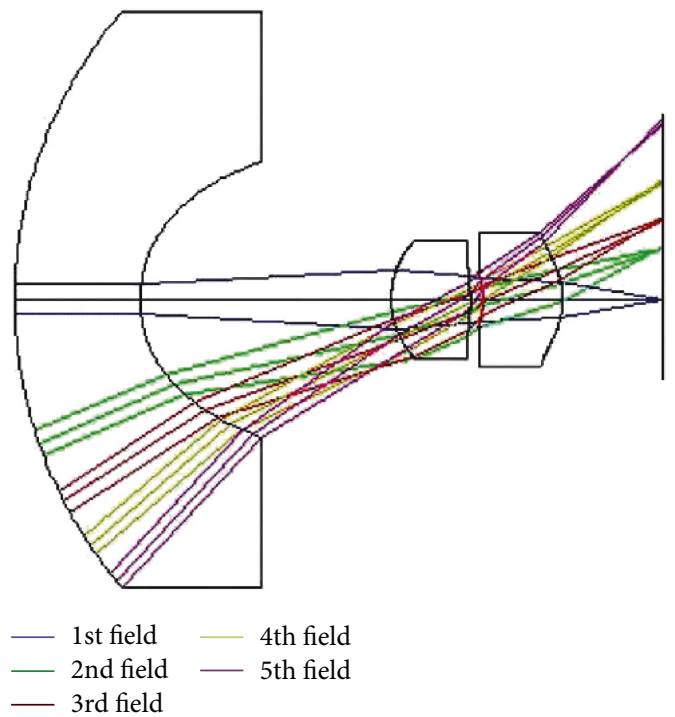

(a)

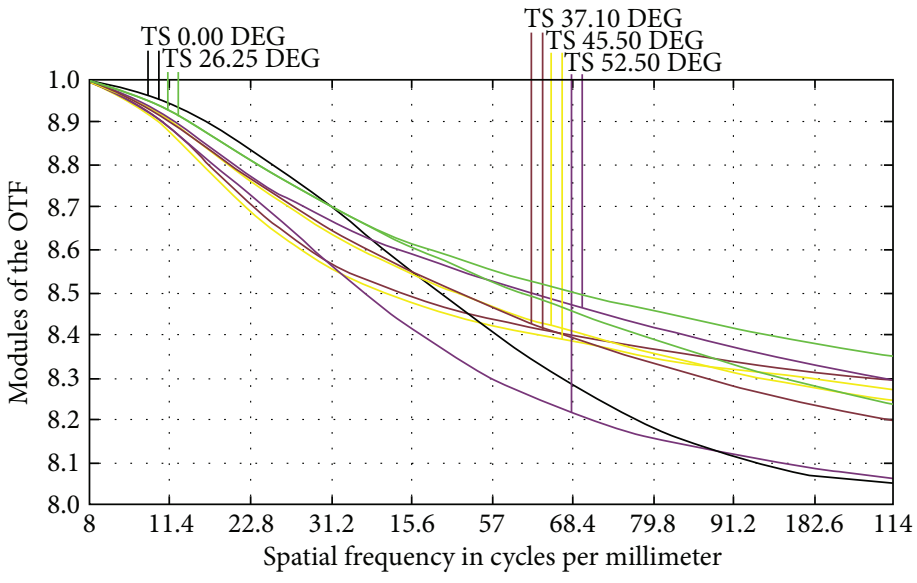

(b)

Figure 5: (a) 3D layout used in existent literature. (b) MTF values generated in existent literature.

TABLE 3: Initial lens parameter table.

\begin{tabular}{|c|c|c|c|c|c|c|}
\hline Surf & Type & Radius (mm) & Thickness (mm) & Glass & Semidiameter $(\mathrm{mm})$ & Conic \\
\hline OBJ & Standard & 15 & 10 & & 10.143393 & 0 \\
\hline 1 & Standard & 75.638249 & 0.228611 & E48R & 1.467268 & 0 \\
\hline 2 & Even Asphere & 0.730586 & 0.375833 & & 0.998383 & -0.7580 \\
\hline 3 & Even Asphere & 0.677934 & 1.181807 & CAF2 & 0.908234 & -1.5743 \\
\hline 4 & Standard & -1.704216 & 0.173513 & & 0.692586 & 0 \\
\hline STO & Standard & Infinity & 0.055548 & & 0.380945 & 0 \\
\hline 6 & Standard & 15.517551 & 0.530639 & CAF2 & 0.441318 & 0 \\
\hline 7 & Standard & -1.085120 & 0.367124 & & 0.619864 & 0 \\
\hline 8 & Even Asphere & -3.065604 & 0.285170 & $\mathrm{COC}$ & 0.714128 & 14.9694 \\
\hline 9 & Even Asphere & 1.970309 & 1.236203 & & 1.097346 & -43.1582 \\
\hline IMA & & Infinity & - & & 2.328690 & 0 \\
\hline
\end{tabular}




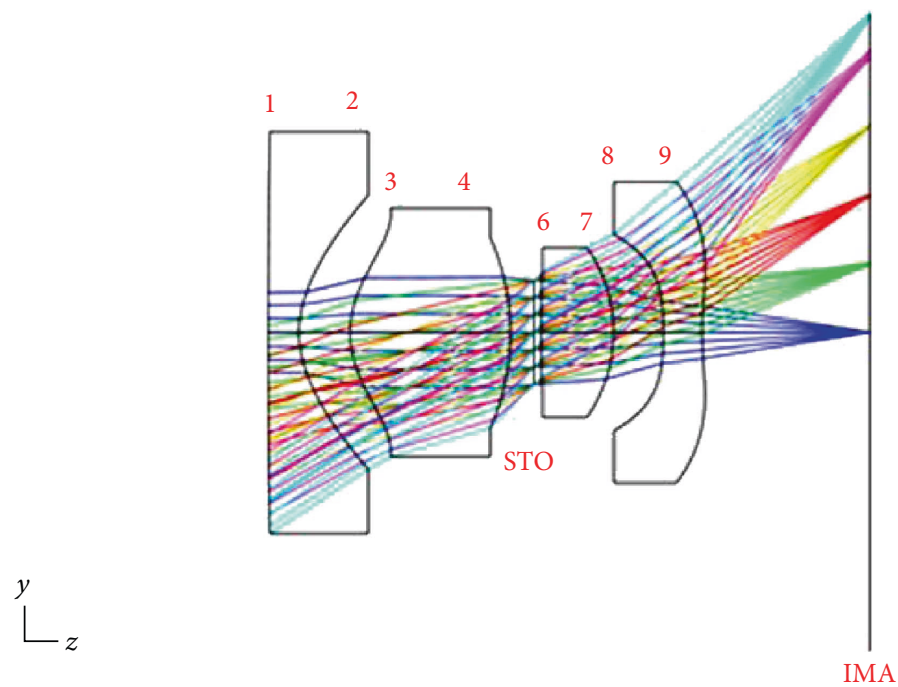

FIgURE 6: 3D image of the initial structure.

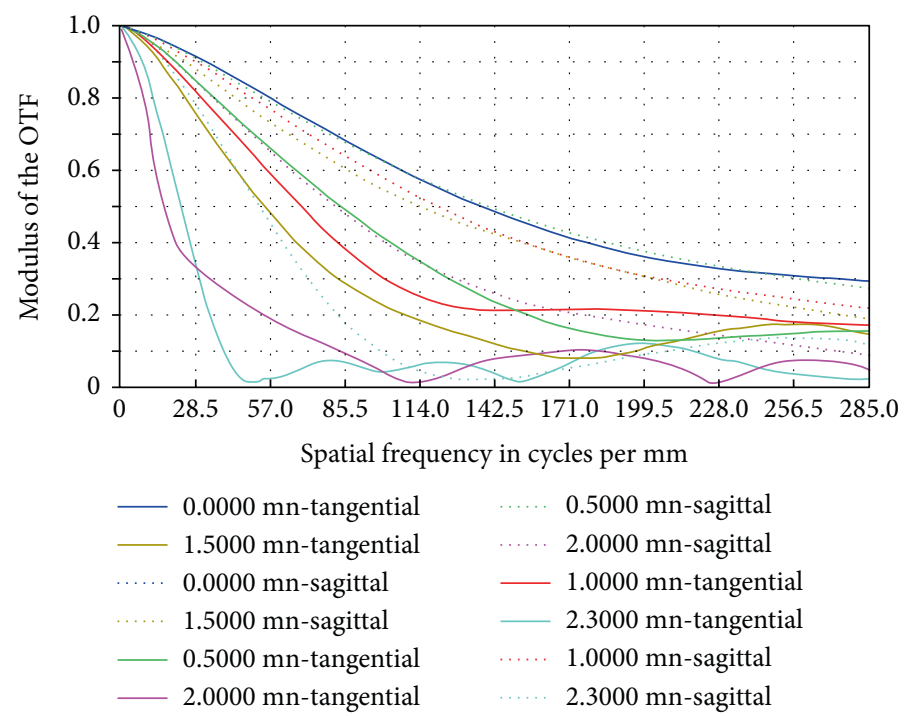

Figure 7: MTF diagram of the initial structure.

The facula diagram shows the magnitude of each light vector on the image plane, as shown in Figure 8. In the diagram, the Airy disk value is $2.161 \mu \mathrm{m}$.

Figure 9 is the ray fan diagram of each FOV, where the left and right diagrams show the light waves radiated on the tangential and sagittal planes, respectively. Light waves that were farther away from the axis produced more optical aberration, whereas those that were closer to the axis generated less optical aberration.

The addition of the fourth lens changed the overall structure, which increased optical aberration and lowered overall optical system performance. In addition, the optical system had a total length of $4.5 \mathrm{~mm}$, which failed to meet the design objective and had to be further optimized to match design requirements.

3.3. Optimized Design. To meet optical design requirements, the initial design structure was optimized using merit functions. ZEMAX design software was employed; it used the damped latest squares algorithm, which involved continuously taking parameter values to diminish the values of the merit functions until the lowest merit function was identified. This method was suitable for parameters that constantly changed. In this study, the merit function was defined as follows:

$$
\mathrm{MF}^{2}=\frac{\sum W_{i}\left(V_{i}-T_{i}\right)^{2}}{\sum W_{i}}
$$

where $W$ is the weight of the operand (in absolute value), $V$ is the current value, $T$ is the target value, and $i$ is the optimization operand number in the merit function table (Table 4).

By using the merit function method, constant optimization was performed until a suitable value was found; the process involved keying in preset operands and their values in 


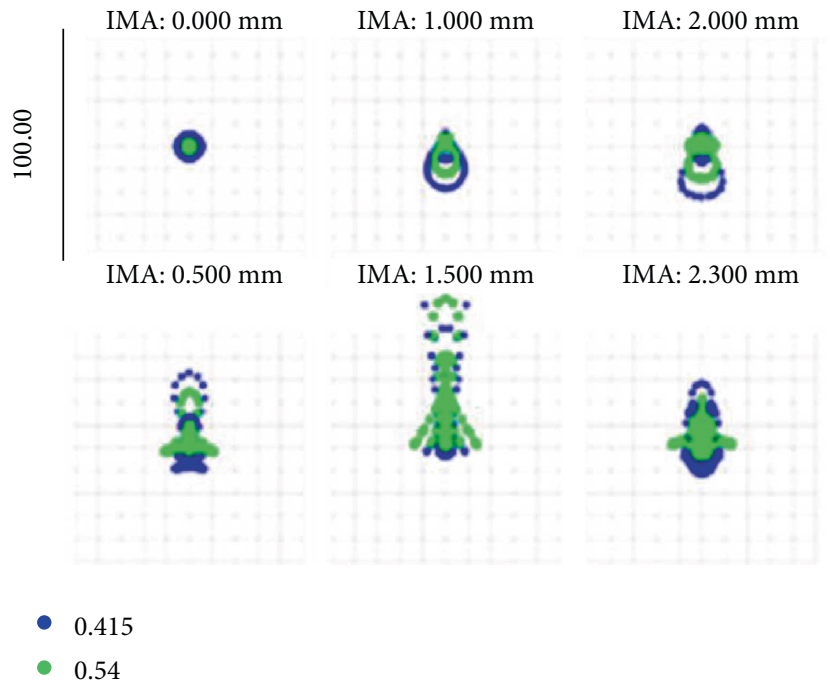

FIGURE 8: Spot diagram of the initial structure.
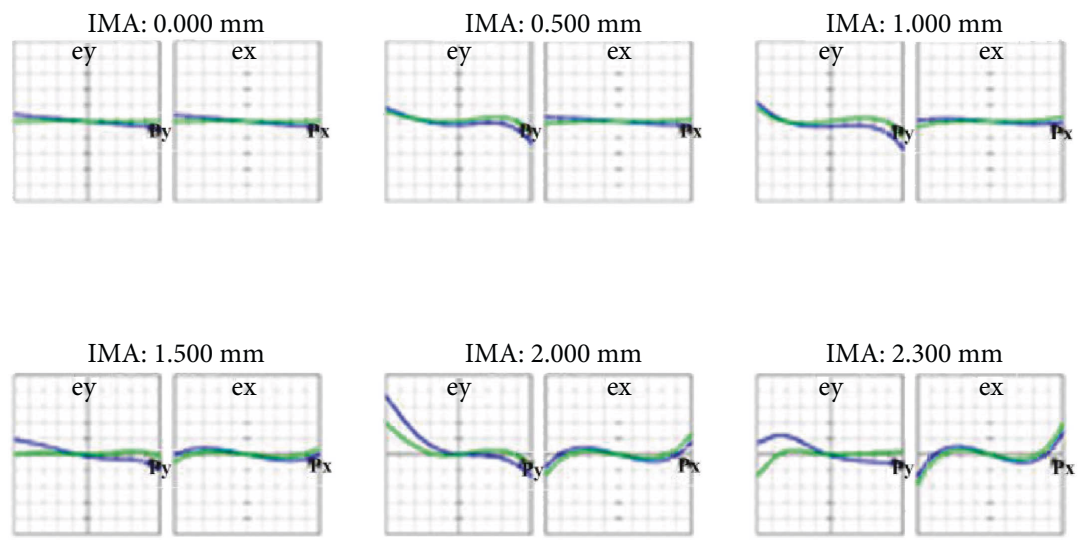

$\begin{array}{ll}- & 0.415 \\ -\quad & 0.540\end{array}$

Figure 9: Ray fan diagram of the initial structure.

the merit function table and complementing it with a built-in operand OPDX (wavefront difference). The optimized initial structure values that were closer to the target values indicated more ideal versions of the camera lens. The optimized lens parameters are shown in Table 5.

3D images before and after optimization are shown in Figure 10 which shows notable improvements in the focal points on the image plane before and after optimization.

Figure 11 shows that at a maximum FOV of 2.3, MTF increased from 0.015 before optimization to 0.13 after optimization, which is $13 \%$ of an ideal image.

The Airy disk values were 2.161 and $2.584 \mu \mathrm{m}$ before and after optimization, as shown in the facula diagram in Figure 12.

Table 6 shows the spot RMS radii before and after optimization, which indicated improvements after optimization.

Figure 13 shows the ray fan diagram, which was primarily used to analyze optical aberrations. The horizontal axis signifies the relative position of the light ray when exiting the pupil, whereas the vertical axis denotes the position of
TABLE 4: Merit function table.

\begin{tabular}{lcc}
\hline Operand & Meaning & Target \\
\hline$T_{1}$ & Effective focal length & $2 \mathrm{~mm}$ \\
$T_{2}$ & Total length & $4 \mathrm{~mm}$ \\
$T_{3}$ & Paraxial image height & $2.3 \mathrm{~mm}$ \\
$T_{4}$ & Relative illumination & $55 \%$ \\
$T_{5}$ & Maximum center thickness of the glass & $1 \mathrm{~mm}$ \\
$T_{6}$ & Minimum center thickness of the glass & $0.3 \mathrm{~mm}$ \\
$T_{7}$ & Maximum edge thickness of the glass & $1 \mathrm{~mm}$ \\
$T_{8}$ & Minimum edge thickness of the glass & $0.3 \mathrm{~mm}$ \\
$T_{9}$ & Distortion max & $15 \%$ \\
\hline
\end{tabular}

the light ray (that has left the pupil) on the image plane. Compared with Figure 9, the results improved substantially for the different FOVs. 
TABLE 5: Lens parameter table after optimization.

\begin{tabular}{|c|c|c|c|c|c|c|}
\hline Surf & Type & Radius (mm) & Thickness (mm) & Glass & Semidiameter $(\mathrm{mm})$ & Conic \\
\hline OBJ & Standard & 15 & 10 & & 8.180505 & 0 \\
\hline 1 & Standard & -2.834516 & 0.278309 & E48R & 0.750946 & 0 \\
\hline 2 & Even Asphere & 1.336097 & 0.031272 & & 0.586826 & 3.335776 \\
\hline 3 & Even Asphere & 0.749830 & 0.464941 & CAF2 & 0.564429 & -0.887225 \\
\hline 4 & Standard & -1.847416 & 0.045157 & & 0.479586 & 0 \\
\hline STO & Standard & Infinity & 0.241786 & & 0.394776 & 0 \\
\hline 6 & Standard & 2.594178 & 0.649424 & CAF2 & 0.623751 & 0 \\
\hline 7 & Standard & -1.139868 & 0.489507 & & 0.716711 & 0 \\
\hline 8 & Even Asphere & -0.810464 & 0.628063 & $\mathrm{COC}$ & 0.737654 & -4.225100 \\
\hline 9 & Even Asphere & -3.836154 & 1.119147 & & 1.327694 & -1.203763 \\
\hline IMA & & Infinity & - & & 2.315860 & 0 \\
\hline
\end{tabular}

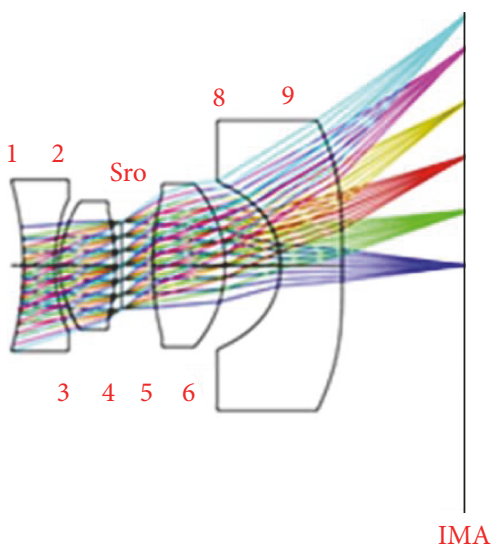

FIGURE 10: 3D image after optimization.

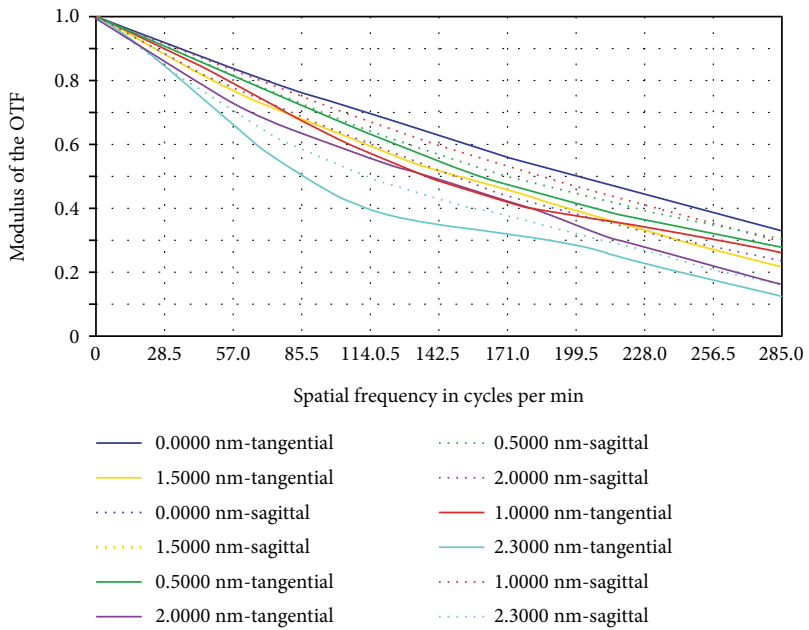

FIgURE 11: MTF after optimization.

Figure 14 shows the curvature of the field, in which the vertical axis represents light waves at different object heights whereas the horizontal axis denotes deviations in the focus positions of paraxial and off-axial rays. The right diagram in Figure 14 is the distortion, in which the vertical axis

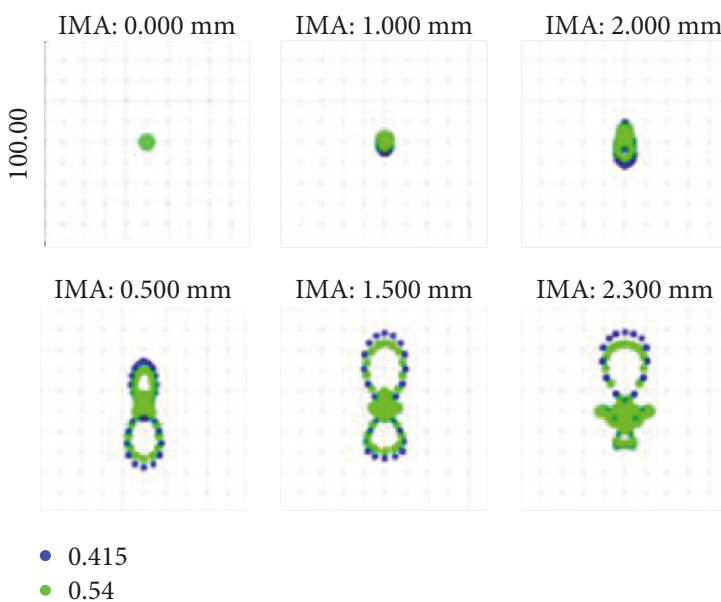

FIGURE 12: Spot diagram after optimization.

TABLE 6: Comparison of RMS radii.

\begin{tabular}{lcc}
\hline Field & Before optimization & After optimization \\
\hline 0.0 & $2.555 \mu \mathrm{m}$ & $1.222 \mu \mathrm{m}$ \\
0.5 & $4.946 \mu \mathrm{m}$ & $2.118 \mu \mathrm{m}$ \\
1.0 & $5.713 \mu \mathrm{m}$ & $4.142 \mu \mathrm{m}$ \\
1.5 & $7.769 \mu \mathrm{m}$ & $8.224 \mu \mathrm{m}$ \\
2.0 & $23.756 \mu \mathrm{m}$ & $10.105 \mu \mathrm{m}$ \\
2.3 & $10.064 \mu \mathrm{m}$ & $10.674 \mu \mathrm{m}$ \\
\hline
\end{tabular}

signifies light waves at different heights whereas the horizontal axis means the differences (measured in percentages) between actual and paraxial rays focused on the image plane. According to the diagram, the size of the distortion was approximately $20 \%$.

Figure 15 shows the relative lighting graph, which yielded a value of approximately 0.61 (i.e., $61 \%$ ).

A comparison between results obtained in this study and those presented in $[9,10]$ is shown in Table 7.

Figure 16 shows the optical system schematic diagram of the internal components of a CE. Compared with traditional 

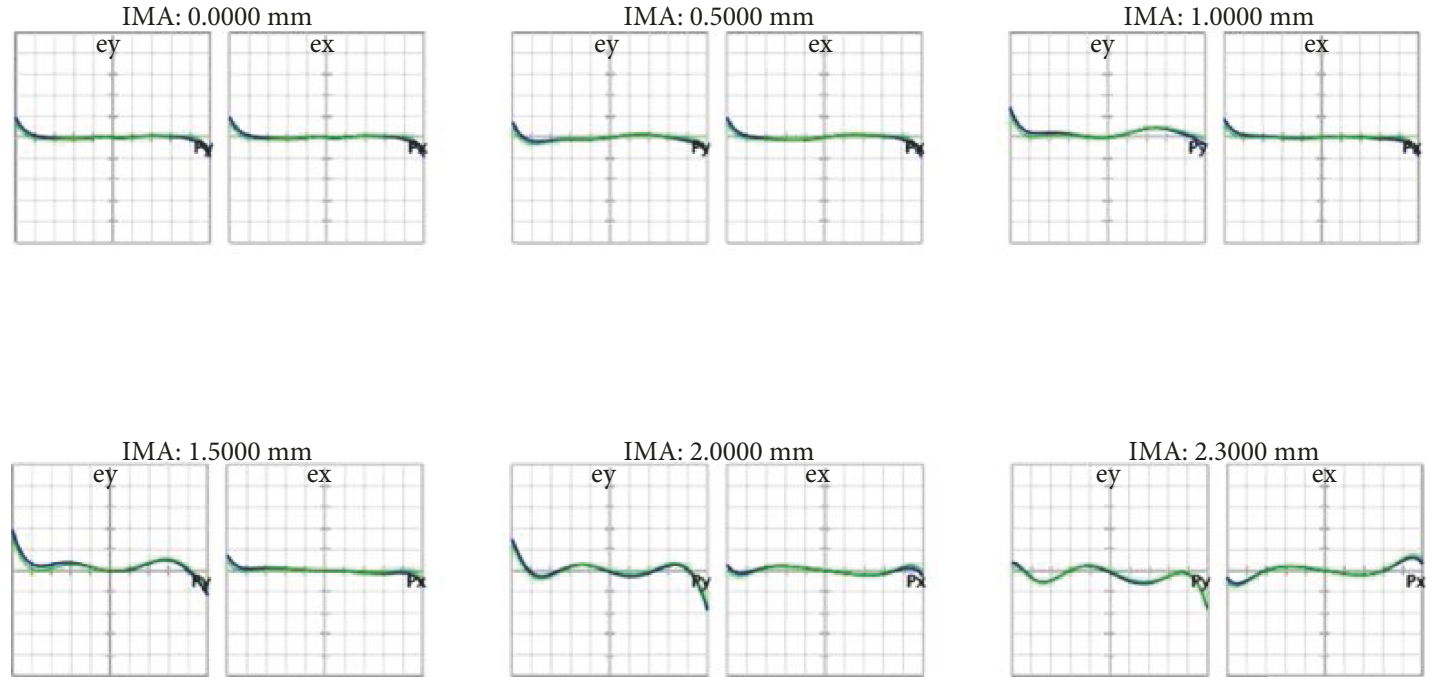

Figure 13: Ray fan diagram after optimization.
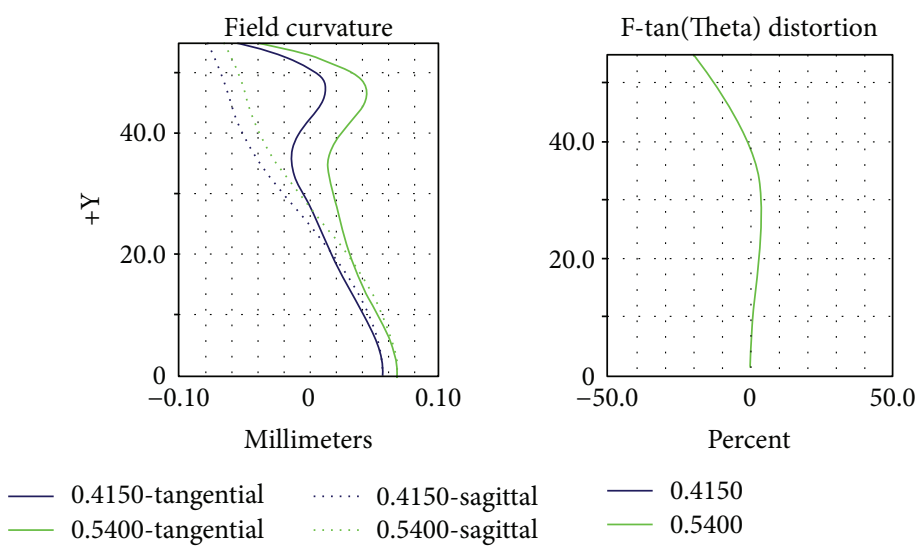

$-0.5400$

Figure 14: Curvature of the field and distortion after optimization.

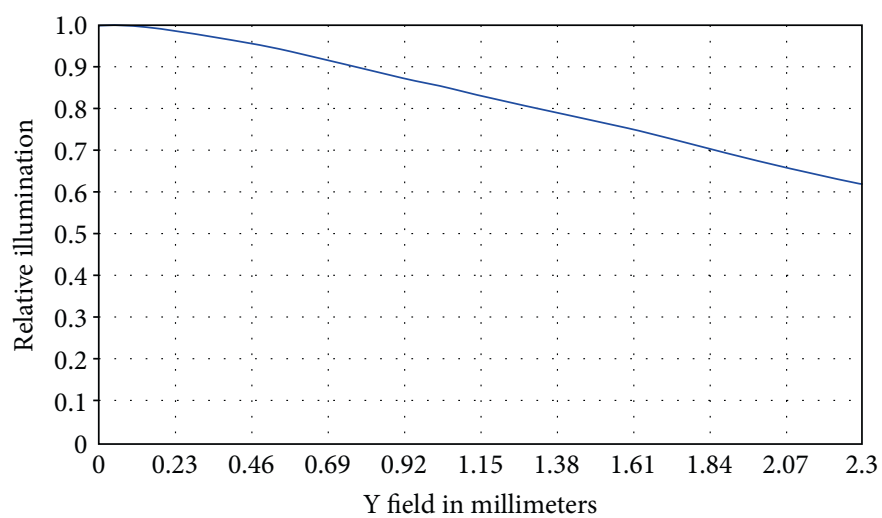

FIGURE 15: Relative lighting after optimization.

CEs, the CE introduced in this study adopted an optical lens design that featured the advantages of small volume, low optical aberration, and high resolution. Comparisons with CEs presented in existent literature show that despite the addition of the fourth lens in this study, the total length of the $\mathrm{CE}$ remained within size restrictions and that the overall FOV increased. As a popular demand for image quality can be expected to intensify in the future, the $\mathrm{CE}$ lens group introduced in this study may serve as a potential option. 
TABLE 7: Comparison between results obtained in this study and those presented in existent literature.

\begin{tabular}{lccc}
\hline & Ou-Yang Mang & Lilai Tang & Result of research \\
\hline Field of view (FOV) & $86^{\circ}$ & $105^{\circ}$ & $109.8^{\circ}$ \\
F/\# & $1 / 2.8$ & $1 / 2.8$ & $1 / 2.8$ \\
Total length & $<3.5 \mathrm{~mm}$ & $<4.5 \mathrm{~mm}$ & $3.94 \mathrm{~mm}$ \\
Relative illumination & $43.55 \%$ & $64 \%$ & $61.7 \%$ \\
Distortion & $<20 \%$ & $15 \%$ & $19.14 \%$ \\
MTF (maximum field) & $53 \%(50 \mathrm{lp} / \mathrm{mm})$ & $40 \%(45 \mathrm{lp} / \mathrm{mm})$ & $34.1 \%(145 \mathrm{lp} / \mathrm{mm})$ \\
CMOS pixels & $26 \%(100 \mathrm{lp} / \mathrm{mm})$ & $20 \%(114 \mathrm{lp} / \mathrm{mm})$ & $12.5 \%(285 \mathrm{lp} / \mathrm{mm})$ \\
\hline
\end{tabular}

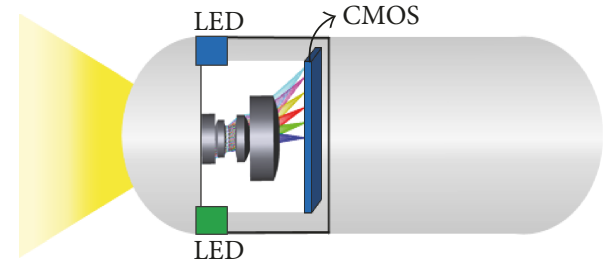

FIGURE 16: CE mechanism.

\section{Conclusions}

Literature has shown that narrow-band technology has made considerable contributions to the detection of pathological changes in human digestive organs and that such technology has been commonly used in clinical experiments. However, the most common usage of narrow-band technology is in electronic endoscopes, and that in the CE is comparatively scant. Therefore, this study develops optical design technology-incorporated narrow-band wavebands in the CEs based on the lens structure of references $[10,11]$.

Although the fourth lens was added to the CE in this study, the total length of the CE remained within $4 \mathrm{~mm}$ and the FOV (obtained through calculations) was approximately $110^{\circ}$, exemplifying a wider viewing angle that meets the study requirements. In general, the design purposes of CEs are higher resolution and larger FOV angle. There are many researches presenting large FOV angles of CEs, but the resolution is not high enough to let the doctors make diagnoses of the disease easily. In simulation results, the distortion was less than $20 \%$ and relative lighting was at least $60 \%$. The MTF values of the CE were $34.1 \%$ and $12.5 \%$ when the spatial frequency was 144 and $285 \mathrm{lp} / \mathrm{mm}$, respectively. This camera lens design is applicable to CMOS image sensors with a size of $1 / 4^{\prime \prime}$, a pixel size of $1.75 \mu \mathrm{m}$, and 3 megapixels. Compared with other CE designs presented in existent literature as shown in Table 7, the CE design introduced in this study displayed superior performance. With the technical advance, the CE image quality can be improved by increasing CMOS resolution and using high-refractive-index material lens in the future.

\section{Disclosure}

The earlier version of the abstract was presented as a poster in 2016 IMETI conference.

\section{Conflicts of Interest}

The authors declare no conflict of interest.

\section{Authors' Contributions}

Chih-Ta Yen performed the simulations and algorithm analysis and contributed to the main part of manuscript writing. Zong-Wei Lai and Yu-Ting Lin contributed to the study conception and the corresponding data analysis. Hsu-Chih Cheng contributed to reviewing the paper and checking the data. All authors contributed to the writing of the paper.

\section{Acknowledgments}

The data analyses were accomplished from Master dissertation, National Formosa University Electrical Engineering Theses \& Dissertations, by Yu-Ting Lin, and the study was also supported in part by the Ministry of Science and Technology of Taiwan MOST 105-2221-E-150-031-.

\section{References}

[1] J. Hou, Y. Zhu, L. Zhang et al., "Design and implementation of a high resolution localization system for in-vivo capsule endoscopy," in 2009 Eighth IEEE International Conference on Dependable, Autonomic and Secure Computing, pp. 209-214, Chengdu, China, 2009.

[2] B. S. Lewis and P. Swain, "Capsule endoscopy in the evaluation of patients with suspected small intestinal bleeding: results of a pilot study," Gastrointestinal Endoscopy, vol. 56, no. 3, pp. 349-353, 2002.

[3] M. Konno, "Capsule endoscope," US Patent 7319896, 2008.

[4] J. M. Geary, Introduction to Lens Design: with Practical Zemax Examples, Willmann-Bell, Richmond, VA, USA, 2002.

[5] S. W. Seo, S. Han, J. H. Seo et al., "MEMS-based liquid lens for capsule endoscope," in Proceedings SPIE 6931, Nanosensors and Microsensors for Bio-Systems 2008, pp. 69310N169310N6, San Diego, CA, USA, 2008.

[6] C. Y. Chen, Design of imaging system in contact capsule endoscope, [M.S. thesis], National Central University Electronic Theses \& Dissertations, 2009.

[7] M. Ou-Yang and W.-D. Jeng, "Design and analysis of radial imaging capsule endoscope (RICE) system," Optics Express, vol. 19 , no. 5, pp. 4369-4383, 2011. 
[8] Y. C. Tseng, H. C. Hsu, P. Han, and C. M. Tsai, "Color multiplexing method to capture front and side images with a capsule endoscope," Applied Optics, vol. 54, no. 28, pp. E241-E248, 2015.

[9] O.-Y. Mang, S.-W. Huang, Y.-L. Chen, C.-H. Lin, T.-Y. Lin, and Y.-T. Kuo, "Distortion improvement of capsule endoscope image," in Proceedings SPIE 6430, Advanced Biomedical and Clinical Diagnostic Systems V, pp. 643018-1-643018-9, Bellingham, WA, USA, 2007.

[10] L. Tang, C. Hu, K. Xie, C. Cheng, and Z. Liu, "Optimized design of capsule endoscopy lens based on ZEMAX," in 2011 IEEE International Conference on Information and Automation, pp. 57-62, Shenzhen, China, 2011.

[11] Taiwan Health Foundation, "The longest organ in the body, small intestine digestion and absorption. GOOD HEALTH," 2009, http://www.twhealth.org.tw/index.php?option=com_zoo \&task=item\&item_id=440\&Itemid $=21$.

[12] Y. T. Lin, A study of optical design with narrow band imaging for capsule endoscope, [M.S. thesis], National Formosa University Electrical Engineering Theses \& Dissertations, Taiwan, 2016.

[13] OmniVISION Technologies, "Color CMOS OV3640 datasheet," October 2008, http://www.uctronics.com/download/ cam_module/OV3640DS.pdf.

[14] Edmund Optics Inc, "Image resource guide," http://www. edmundoptics.com.tw/resources/application-notes/imaging/ resolution/.

[15] Y. Sano, Y. Saito, K. I. Fu et al., "Efficacy of magnifying chromoendoscopy for the differential diagnosis of colorectal lesions," Digestive Endoscopy, vol. 17, no. 2, pp. 105-116, 2005.

[16] H. Machida, Y. Sano, Y. Hamamoto et al., "Narrow-band imaging in the diagnosis of colorectal mucosal lesions: a pilot study," Endoscopy, vol. 36, no. 12, pp. 1094-1098, 2004.

[17] P. Lukes, M. Zabrodsky, J. Plzak et al., "Narrow band imaging (NBI)," in Endoscopic Method for Detection of Head and Neck Cancer, Endoscopy, S. Amornyotin, Ed., InTech, 2013, https:// www.intechopen.com/books/endoscopy/narrow-bandimaging-nbi-endoscopic-method-for-detection-of-head-andneck-cancer. 


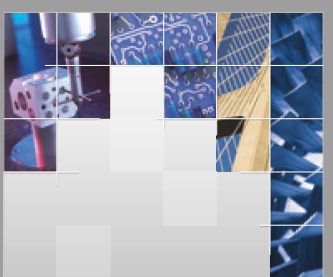

\section{Enfincering}
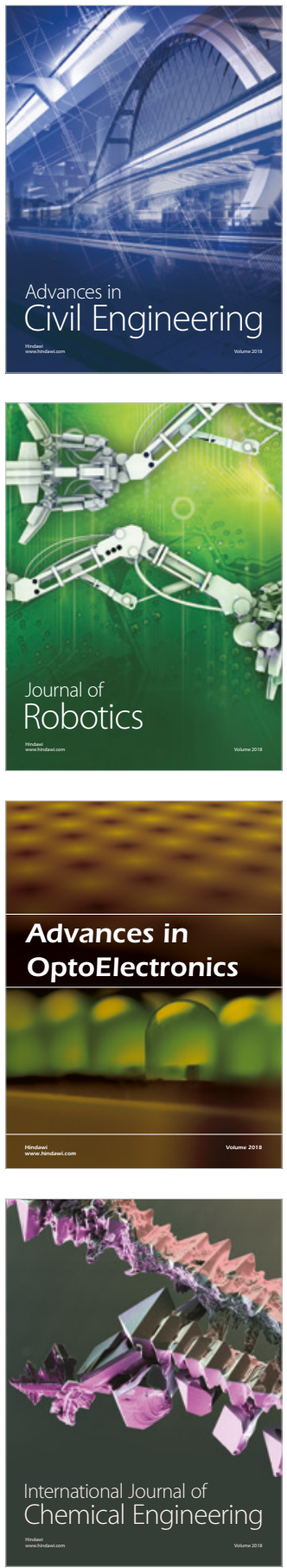

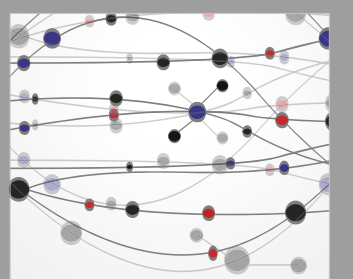

\section{Rotating \\ Machinery}

The Scientific World Journal

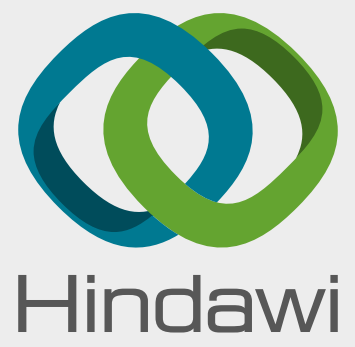

Submit your manuscripts at

www.hindawi.com
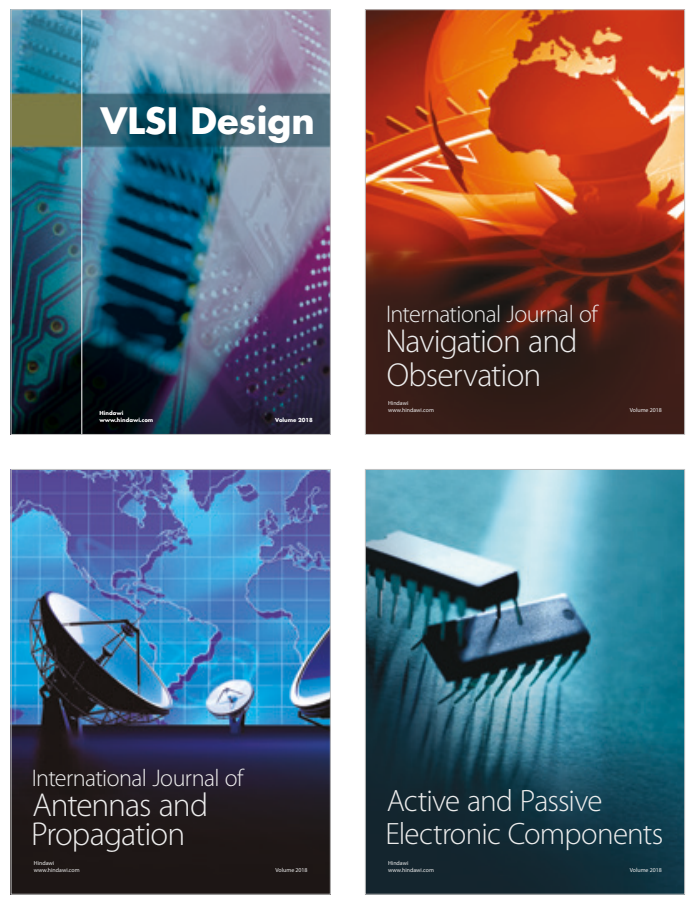
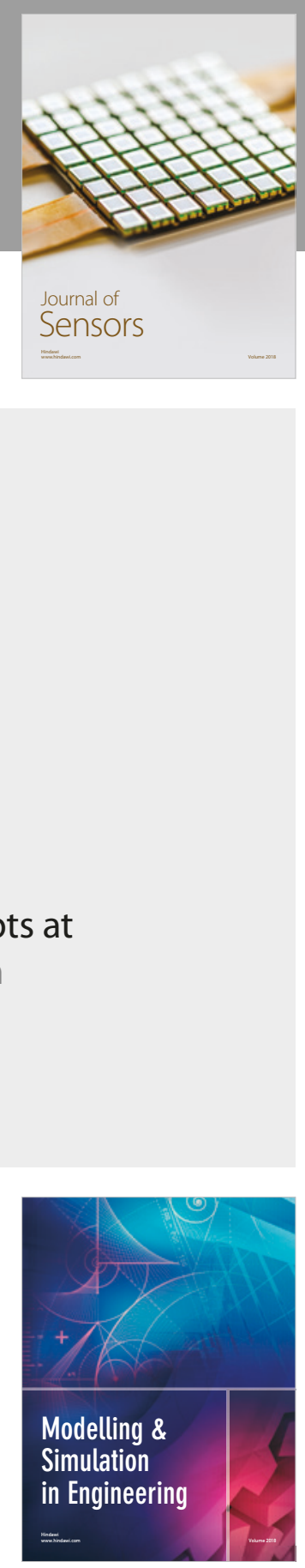

\section{Advances \\ Multimedia}
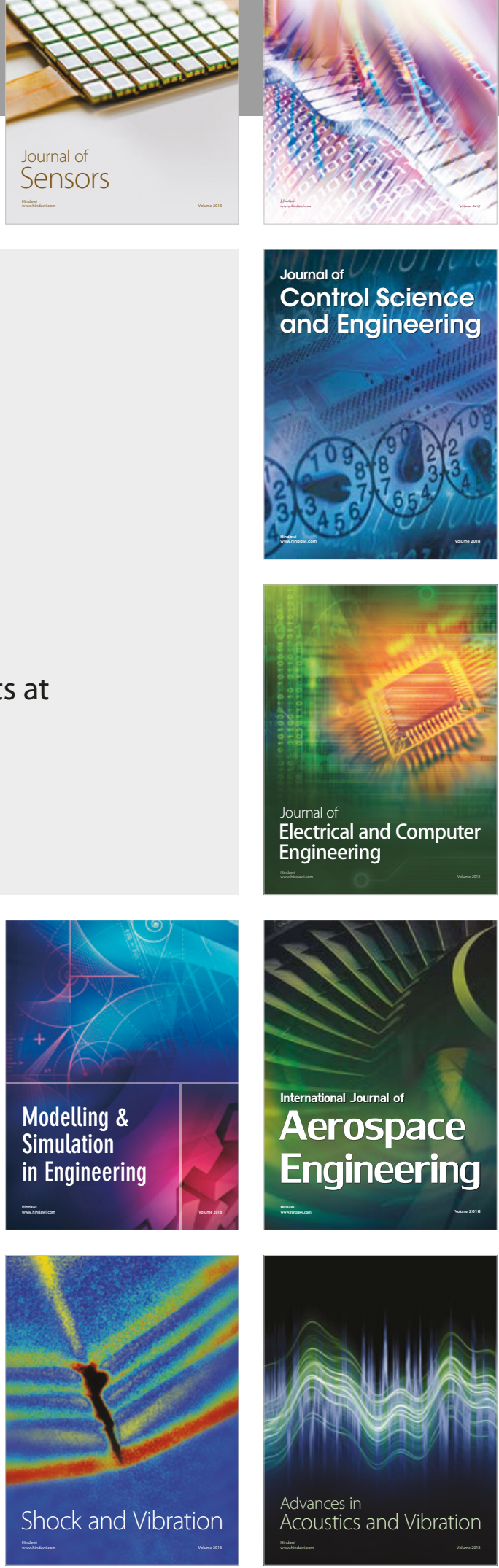